\title{
Decolonization of orthopedic surgical team $S$. aureus carriers: impact on surgical-site infections
}

\author{
M. Portigliatti Barbos • Barbara Mognetti • \\ S. Pecoraro $\cdot$ W. Picco $\cdot$ V. Veglio
}

Received: 26 June 2009/ Accepted: 10 January 2010/Published online: 30 January 2010

(C) Springer-Verlag 2010

\begin{abstract}
Background Orthopedic surgical-site infection (SSI), mostly due to $S$. aureus, is recognized as a major adverse event. This research aims to verify the usefulness of surgical team decolonization in order to reduce the risk of surgical-site infection.

Materials and methods We performed swabs of both nares and oropharynx to identify $S$. aureus carriers among orthopedic team members who consented to cooperate with the study. Carriers were treated with local application of mupirocin ointment.

Results Retrospective study of 1,000 consecutive patients operated before surgical team decolonization showed $6 \%$ SSIs. Of the 300 cases considered after decolonization, none developed SSI.

Conclusions Though we are aware that more data need to be collected, this work might be relevant for the introduction of a new preventive protocol.
\end{abstract}

Keywords Surgical-site infections $\cdot$ S. aureus .

Prevention · Orthopedic surgery

\section{Introduction}

Surgical-site infections (SSIs) are a major adverse event for patients and their surgeons. Orthopedic SSIs cause

\footnotetext{
M. Portigliatti Barbos · B. Mognetti ( $\varangle)$

Department of Clinical and Biological Sciences,

University of Turin, Regione Gonzole 10,

10043 Orbassano (To), Italy

e-mail: barbara.mognetti@unito.it

S. Pecoraro - W. Picco - V. Veglio

Fondazione Pinna Pintor, Turin, Italy
}

substantial morbidity, prolonging hospital stay by a median of 2 weeks, doubling rehospitalization rates, and more than tripling overall healthcare costs [1]. Staphylococcus aureus is the most important pathogen after surgical procedures, and its nasal carriage is the main risk factor for SSI caused by $S$. aureus [2], since carriers are two to nine times more likely to acquire $S$. aureus SSIs than noncarriers [3]. The mean carriage rate in the general population is $37.2 \%$ [3]. Several studies have been conducted on nasal decolonization in patients undergoing orthopedic surgery by using perioperative intranasal mupirocin $[4,5]$, an attractive prevention strategy, being a safe and simple method to eradicate nasal colonization. Preoperative patient decolonization significantly decreases incidence of $S$. aureus SSIs $[4,5]$. Nevertheless, no experience is documented regarding surgical team screening for $S$. aureus carriers and the use of perioperative mupirocin for decolonization in order to extend the prophylaxis against $S$. aureus orthopedic SSIs. Therefore, the focus of this study is the role of the surgical team as a possible risk factor for SSI and to suggest a suitable and comprehensive protocol for prevention.

\section{Materials and methods}

In order to establish the frequency of $S$. aureus nasal carriers among orthopedic surgical team members operating within $2 \mathrm{~m}$ from the surgical site, we performed swabs of both nares and oropharynx on 75 workers of the operating theater (orthopedic surgeons, anesthesiologists and nurses) who provided informed consent to be enrolled in the study.

Each collection swab was streaked onto S. aureusselective mannitol salt agar plates, incubated for $24 \mathrm{~h}$ at $37^{\circ} \mathrm{C}$, and examined for growth. 
According to swab results, carriers were treated with daily nasal application of mupirocin ointment (Glaxo) for 5 days. The treatment was repeated 6 months later. Results of mupirocin treatment were checked 3 months after treatment by repeating swabs of both nares and oropharynx.

SSIs in both pre- and posttreatment groups were diagnosed through wound swab and cultural examination in presence of altered clinical and biohumoral parameters.

\section{Results}

Thirty out of 75 (40\%) swabs resulted positive for presence of $S$. aureus.

Susceptibility testing on the isolated bacteria indicated that, while none of the carriers was mupirocin resistant, 23/30 produced $\beta$-lactamase (therefore being penicillin resistant) and 5/30 were oxacillin resistant (Fig. 1).

Despite mupirocin application, one surgeon appeared positive 3 months after treatment, and was therefore excluded from the study.

In a second step of this study we compared the rate of S. aureus SSIs in two series of patients operated before (1,000 patients, retrospective analysis) and after (300 patients) decolonization of surgical teams.

All 75 team members, both carriers and noncarriers, had to be considered because of the variable composition of the surgical teams. Retrospective study on 1,000 consecutive patients operated before nasal decolonization of the surgical team showed 6\% SSIs. Of the 300 cases considered after nasal decolonization, none developed SSI ( $p=0.439$ Kruskall-Wallis test).

Operations were distributed as presented in Table 1. The two considered groups are equivalent.

\section{Discussion}

This preliminary study shows for the first time how a prophylactic intervention on the surgical team can decrease the SSI rate. Though we are aware that various problems still have to be solved (such as intermittent carriers and possible false negatives, the duration of decolonization efficacy and the appearance of resistance to treatment) and that more data need to be collected to reinforce statistical analysis, we consider this prophylactic approach to be promising and worth being introduced into daily surgical practice.

In accordance with published statistics [3], our work confirms the presence of $40 \% \mathrm{~S}$. aureus carriers in surgical teams, thus emphasizing the importance of decolonization treatment not only for patients but-probably even more strikingly_for health workers in the operating theater. According to the CDC Guideline for Prevention of SSI, healthcare organizations have to implement policies to prevent transmission of microorganisms from personnel to patients [6]. This work might be relevant for the development of preventive protocols and guidelines for the introduction of this kind of prophylaxis to reduce SSIs.
Fig. 1 Percentage of $S$. aureus carriers in the screened population of orthopedic surgical team members (a) and antibiotic resistance in the isolated bacterial colonies (b) (a) S. aureus presence in nasal and oropharyngeal swabs

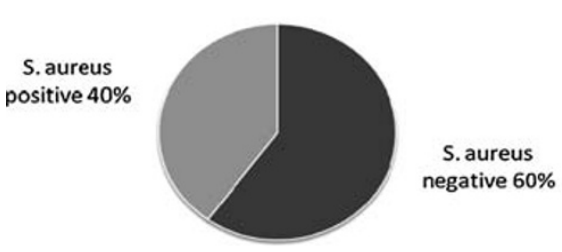

(b) antibiogram results of positive nasal and oropharyngeal swabs

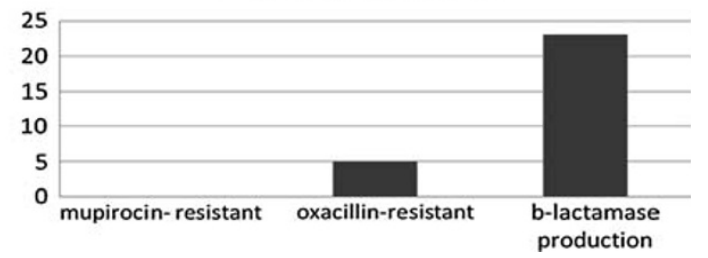

Table 1 Distribution of operations within the two considered groups

Values are expressed as approximate number and as percentage. Groups are equivalent in composition

\begin{tabular}{|c|c|c|c|c|}
\hline & \multicolumn{2}{|c|}{ Pretreatment } & \multicolumn{2}{|c|}{ Posttreatment } \\
\hline & Number & Percentage $(\%)$ & Number & Percentage $(\%)$ \\
\hline Hip-knee prosthesis & 40 & 4 & 13 & 4.3 \\
\hline Shoulder-knee arthroscopy & 515 & 51.5 & 167 & 55.7 \\
\hline Foot surgery & 69 & 6.9 & 26 & 8.7 \\
\hline Hand surgery & 210 & 21 & 67 & 22.3 \\
\hline $\begin{array}{l}\text { Anterior cruciate ligament } \\
\text { reconstruction }\end{array}$ & 61 & 6.1 & 20 & 6.7 \\
\hline Other & 105 & 10.5 & 7 & 2.3 \\
\hline Total & 1,000 & & 300 & \\
\hline
\end{tabular}


Acknowledgments Authors thank Glaxo for providing mupirocin, and Prof. Plinio Pinna Pintor, the Pinna Pintor Foundation, and Humanitas for encouraging and supporting this research. The study was performed in accordance with the Declaration of Helsinki and it was approved by the local ethical committee.

Conflict of interest statement The authors declare that they have no conflict of interest related to the publication of this article.

\section{References}

1. Whitehouse JD, Friedman ND, Kirkland KB et al (2002) The impact of surgical-site infections following orthopedic surgery at a community hospital and a university hospital: adverse quality of life, excess length of stay, and extra cost. Infect Control Hosp Epidemiol 23:183-189
2. Kalmeijer MD, van Nieuwland-Bollen E, Bogaers-Hofman D et al (2000) Nasal carriage of Staphylococcus aureus is a major risk factor for surgical-site infections in orthopedic surgery. Infect Control Hosp Epidemiol 21:319-323

3. Kluytmans J, van Belkum A, Verbrugh H (1997) Nasal carriage of Staphylococcus aureus: epidemiology, underlying mechanisms, and associated risks. Clin Microbiol Rev 10:505-520

4. Wilcox MH, Hall J, Pike H et al (2003) Use of perioperative mupirocin to prevent methicillin-resistant Staphylococcus aureus (MRSA) orthopaedic surgical site infections. J Hosp Infect 54:196-201

5. Gernaat-van der Sluis AJ, Hoogenboom-Verdegaal AM, Edixhoven PJ et al (1998) Prophylactic mupirocin could reduce orthopedic wound infections. 1044 patients treated with mupirocin compared with 1260 historical controls. Acta Orthop Scand 69:412-414

6. Mangram AJ, Horan TC, Pearson ML et al (1999) Guideline for prevention of surgical site infection, 1999. Hospital Infection Control Practices Advisory Committee. Infect Control Hosp Epidemiol 20:250 